\title{
A Cdc2-related protein kinase hPFTAIRE1 from human brain interacting with 14-3-3 proteins
}

\author{
Yankun Gao ${ }^{1}$, Mei Jiang ${ }^{1}$, Tao Yang ${ }^{1}$, Jian $\mathrm{Ni}^{1}$, Jiangye Chen ${ }^{1}$ \\ ${ }^{1}$ State Key Laboratory of Molecular Biology, Institute of Biochemistry and Cell Biology, Shanghai Institutes for Biological Sciences, \\ Chinese Academy of Sciences, 320 Yue-yang Road, Shanghai 200031, China
}

hPFTAIRE1 (PFTK1), a Cdc2-related protein kinase, is highly expressed in human brain. It exhibits cytoplasmic distribution in Hela cells, although it contains two nuclear localization signals (NLSs) in its N-terminus. To search for its substrates and regulatory components, we screened a two-hybrid library by using the full-length hPFTAIRE1 as a bait. Four 14-3-3 isoforms $(\beta, \varepsilon, \eta, \tau)$ were identified interacting with the hPFTAIRE1. We found a putative 14-3-3 binding consensus motif (RHSSPSS) in the hPFTAIRE1, which overlapped with its second NLS. Deletion of the RHSSPSS motif or substitution of $\mathrm{Ser}^{119}$ with Ala in the conserved binding motif abolished the specific interaction between the hPFTAIRE1 and the 14-3-3 proteins. The mutant S120A hPFTAIRE1 also showed a weak interaction to the 14-3-3 proteins. The results suggested that the $\mathrm{Ser}^{119}$ is crucial for the interaction between hPFTAIRE1 and the 14-3-3 proteins. All the hPFTAIRE1 mutants distributed in cytoplasm of Hela cells and human neuroblastoma cells (SH-SY5Y) when fused to the C-terminus of a green fluorescent protein (GFP), indicating that binding with the 14-3-3 proteins does not contribute to the subcellular localization of the hPFTAIRE1, although the binding may be involved in its signaling regulation.

Cell Research (2006) 16:539-547. doi:10.1038/sj.cr.7310071; published online 15 June 2006

Keywords: hPFTAIRE1, 14-3-3 proteins, 14-3-3 binding motif, two-hybrid

\section{Introduction}

The Cdks (Cyclin-dependent kinases) are serine/threonine kinases that had been shown to be key players in the control of cell cycle progression [1]. These various Cdks share high similarity in their amino acid sequence, and contain a PSTAIRE motif for binding of their cyclin partners. Over the last few years, a number of kinases have been identified in mammalian based on structural similarity to $\mathrm{p} 34^{\mathrm{cdc} 2}[2]$, and their cyclin partners remain to be identified. These Cdc2-related protein kinases derived their names from the presence of an amino acid substitution for serine in the cyclin binding sequence PSTAIRE. Some of these Cdc2-related kinases, such as PFTAIRE, PCTAIRE, PITSIRE and Cdk5, have been shown to play important

Correspondence: Jiangye Chen

Tel: 86-21-54921251; Fax: 86-21-54921011

E-mail:jychen@sunm.shcnc.ac.cn

Received 22 Apr 2005; revised 28 Mar 2006; accepted 11 Apr 2006; published online 15 Jun 2006 roles during cell division, gametogenesis and differentiation [3-6]. Two mouse PFTAIRE (PFTAIRE and mPFTAIRE1) genes have been reported $[7,8]$. The mPFTAIRE1 has a 46 amino acid extension than the PFTAIRE at its N-terminus. The PFTAIRE is expressed primarily in the postnatal and adult nervous system by in situ hybridization and indirect immunofluorescence. In neurons, PFTAIRE protein was localized in the nucleus and cytoplasm of cell [7]. The mPFTAIRE 1 is ubiquitously expressed in murine tissues and highly expressed in brain, testis and embryo. The mPFTAIRE1 is implicated in the process of meiosis as well as neuron differentiation. We have isolated a Cdc2-related protein kinase PFTAIRE1 from human (hPFTAIRE1) [9]. The hPFTAIRE1 was highly expressed in brain, pancreas, kidney, heart, testis and ovary. Although the hPFTAIRE1 contains two potential nuclear localization signals (NLS) at its N-terminus, GFP-hPFTAIRE1 fusion protein expressed in Hela cells distributed mainly in cytoplasm. To search its substrate and explore the cytoplasmic distribution mechanism, we screened an adult human brain two hybrid cDNA library by using the hPFTAIRE1 as a bait and identified 
several hPFTAIRE1 interacting proteins, including septin family member KIAA0202 [10] and four 14-3-3 isoforms $(\beta, \varepsilon, \eta, \tau)$.

14-3-3 proteins are a family of highly conserved acidic proteins, which are expressed ubiquitously in all eukaryotic cells, especially abundant in brain cells and other CNS cells. Seven 14-3-3 isoforms encoded by seven distinct genes are identified in mammalian cells. More than 10 isoforms are identified in plants, and two isoforms are identified in yeast, Drosophila, and Caenorhabditis elegans [11, 12]. The 14-3-3 proteins are mainly localized in cytoplasm. More and more signaling proteins have been reported as 14-3-3 ligands. This broad range of partners suggests a role for 14-3-3 as general biochemical regulators. Through interaction with its effector proteins, 14-3-3 participates in the regulation of diverse biological processes, including neuronal development, cell growth control, apoptosis, and viral and bacterial pathogenesis $[13,14]$. Research work on nuclear proteins such as Cdc 25 and NFAT suggests that 14-3-3 binding is also involved in intracellular targeting. 14-3-3 binding keeps these nuclear proteins in the cytoplasm $[15,16]$. Many serine/threonine kinases are capable of generating 14-3-3 binding sites, including Raf-1, protein kinase $\mathrm{A}$ and protein kinase $\mathrm{C}$. The most important feature of the 14-3-3 proteins is their capability of recognizing and binding to certain motifs containing phosphoserine. By extensive screening of phosphoserine-orientated peptide libraries, it is known that there are mainly two 14-3-3 binding consensus motifs: $\mathrm{RSxpSxP}$ and $\mathrm{Rx}_{1-2} \mathrm{Sx}_{2-3} \mathrm{~S}$ [17]. By interaction with more than 100 binding partners, 14-3-3 proteins modulate the action of proteins that are involved in cell cycle and transcriptional control, signal transduction, intracellular trafficking and regulation of ion channels.

In this study, we screened hPFTAIRE1 interacting proteins in two hybrid system and identified four 14-3-3 isoforms $(\beta, \varepsilon, \eta, \tau)$. The association of the hPFTAIRE1 with 14-3-3 proteins and their intracellular targeting mechanism were analyzed.

\section{Materials and Methods}

\section{Plasmid construction}

The full open reading frame of $h P F T A I R E 1$ gene was inserted into $E c o R$ I and BamH I sites of pGilda vector to generate pGilda-PFT and used as a bait plasmid for two-hybrid screening. The hPFTAIRE1 coding region was inserted into $E c o R$ I and $X h o$ I sites of pcDNA3-myc to construct pcDNA3-PFT-myc for co-immunoprecipitation assay. All constructed plasmids were confirmed by DNA sequencing.

\section{Yeast two-hybrid assay}

A human adult brain LexA two-hybrid cDNA library (Clontech) in which cDNA fragments inserted in pB42AD was used for twohybrid screening. pGilda-PFT (LexA-PFTAIRE1 fusion) was used as a bait to screen the library. All the procedures were followed the protocol from Clontech.

The pGilda-PFT and library plasmids were sequentially transformed into yeast host strain CJY151 (MATa, his3, $\left.\operatorname{trp} 1, \operatorname{Lex} A_{o p(i A 6)}\right)^{-}$ leu2, LexA $A_{o p(i A 8)-l a c Z}$ ) [18]. A total of $1 \times 10^{8}$ transformants were plated onto the yeast galactose drop-out minimal medium lacking of histidine, tryptophan and leucine, incubated for 3 days at $30{ }^{\circ} \mathrm{C}$. The $\mathrm{Leu}^{+}$colonies were collected and assayed for $\beta$-galactosidase activity. The library plasmids from the positive colonies ( $\mathrm{Leu}^{+}$and $\beta-\mathrm{Gal}^{+}$) were confirmed by PCR based restriction map and DNA sequence analysis.

\section{Mutagenesis and $\beta$-galactosidase activity assay}

Three hPFTAIRE1 mutants: hPFTAIRE1D (hPFTAIRE1 with $\mathrm{S}^{119}$ SPSS 5 residues deleted), S119AhPFTAIRE1 (hPFTAIRE1 with Ser119 $\rightarrow$ Ala substitution) and S120AhPFTAIRE1 (hPFTAIRE1 with Ser120 $\rightarrow$ Ala substitution) were constructed by PCR amplification using Mutagenesis kit (Strategene). The hPFTAIRE1 mutants were constructed into pGilda, pcDNA3 and pEGFPC2. All plasmids were confirmed by sequencing. Various pairs of the pGilda-PFT mutants and $\mathrm{pB} 42 \mathrm{AD}-14-3-3$ plasmids were cotransformed into yeast reporter strain CJY151 ( MATa, his3, trp1,LexA op(iÁ) $_{\text {-leu2, LexA }}$ op(iAs)-lacZ ) [18] to test for the $\beta$-galactosidase activity. $\beta$-galactosidase assays with X-gal as substrate was performed as described (MATCHMAKERTwo-Hybrid System, Clontech).

\section{Co-immunoprecipitation assay and Western blot analysis}

Hela cell extracts were prepared by homogenizing cultured cells in CelLytic $M$ cell lysis reagent(Sigma). Insoluble materials were pelleted by centrifugation at $12000 \times \mathrm{g}$ for $15 \mathrm{~min}$ at $4{ }^{\circ} \mathrm{C}$. Protein concentration was determined using a Bio-Rad DC protein assay kit (Bio-Rad). Western blot analysis was conducted the same as previously described [18]. Appropriate antibodies were used to detect the corresponding proteins according to Sambrook [19]. The anti-HA, anti-LexA, anti-myc, anti-14-3-3 $\beta$ and anti-GFP antibodies were purchased from Santa cruz. The mouse IgG was purchased from Sigma. The hPFTAIRE1 was expressed and purified from bacteria (data not shown). The rabbit polyclonal anti-PFTAIRE1 antibody was made by antibody research center in Shanghai Institutes for Biological Sciences, CAS.

\section{Subcellular localization}

Hela cells and SH-SY5 cells were seeded on coverslips and transiently transfected with $5 \mu \mathrm{g}$ of pEGFP-C2 (Clontech), pEGFPC2-PFT, pEGFPC2-PFTD, pEGFPC2-S119APFT and pEGFPC2S120APFT respectively using Lipofectamine reagent (Gibco BRL). 48 hours after transfection, cells were washed three times with PBS, fixed $20 \mathrm{~min}$ in $4 \%$ paraformaldehyde, washed three times again with PBS. To visualize nuclei, cells were treated with $0.1 \%$ triton X-100 and incubated in $1 \mathrm{mg} / \mathrm{ml} \mathrm{4,} \mathrm{6-diamidino-2-phenylindole} \mathrm{(DAPI)} \mathrm{in}$ phosphate-buffed saline.

\section{Results}

Interactions between the hPFTAIRE1 and four 14-3-3 isoforms $(\beta, \varepsilon, \eta, \tau)$

The hPFTAIRE1 encodes for a Cdc2-related protein kinase that is highly expressed in human brain [9]. To search for its regulatory substrates an adult human brain 
Lex-A two-hybrid library was used to screen for its interacting proteins. A full-length hPFTAIRE1 was used as a bait. Among $1 \times 10^{8}$ colonies screened, 243 colonies could grow on the minimal medium, 81 of them showed a positive response in the $\beta$-galactosidase assay. These 81 inserts represent 7 different cDNA fragments, 4 of them encoding for four 14-3-3 isoforms $(\beta, \varepsilon, \eta, \tau)$. All the cDNA inserts from $\mathrm{pB} 42 \mathrm{AD}-14-3-3$ plasmids encoded for full open reading frame of 14-3-3 proteins which fused with 88-residues acidic activator B42 and a hemagglutinin (HA) epitope tag in frame. The cDNA inserts for 14-3-3 $\beta, 14-3-3 \varepsilon$ and 14$3-3 \tau$ are $1 \mathrm{~kb}$ in length and that for $14-3-3 \eta$ is $2 \mathrm{~kb}$ which contains a longer 3 '-non coding region.

The interactions between hPFTAIRE1 and the four 14-3-3 isoforms were verified by two hybrid analysis. Cotransforming pGilda-PFT and pB42AD-14-3-3 plasmids into the yeast CJY151 showed $\beta$-galactosidase activity in $3 \mathrm{~h}$ incubation at $30{ }^{\circ} \mathrm{C}$ (Figure $1 \mathrm{~A}$ ). The pGilda-PFT or pB42AD-14-3-3 plasmids co-transformed with control vectors did not detect any $\beta$-galactosidase activity. The interaction between the hPFTAIRE1 and the four 14-3-3 isoforms can also promote the expression of LEU2 gene when it is used as a reporter, all the co-transformed strains grow well in leu' medium (Figure 1B). The physical interactions between the hPFTAIRE1 and the four 14-3-3 isoforms were confirmed by co-immunoprecipitation in yeast cells. LexA-hPFTAIRE1 was co-expressed separately with four HA-14-3-3 proteins in CJY151. The yeast lysates were immunoprecipitated by anti-LexA antibody and then detected with anti-HA antibody using Western blot. A 35 $\mathrm{kDa}$ band which corresponds to 14-3-3 was detected in the pGilda-PFT and pB42AD-14-3-3 co-transformed cells, and the control showed a negative result (Figure 1C).

Since the 14-3-3 proteins are ubiquitously expressed in all eukaryotic cells and hPFTAIER 1 is also expressed in several cell lines tested (data not shown). Therefore Hela cells were used to confirm the interaction between hPFTAIRE1 and 14-3-3 proteins in vivo. The immuno-

A

B
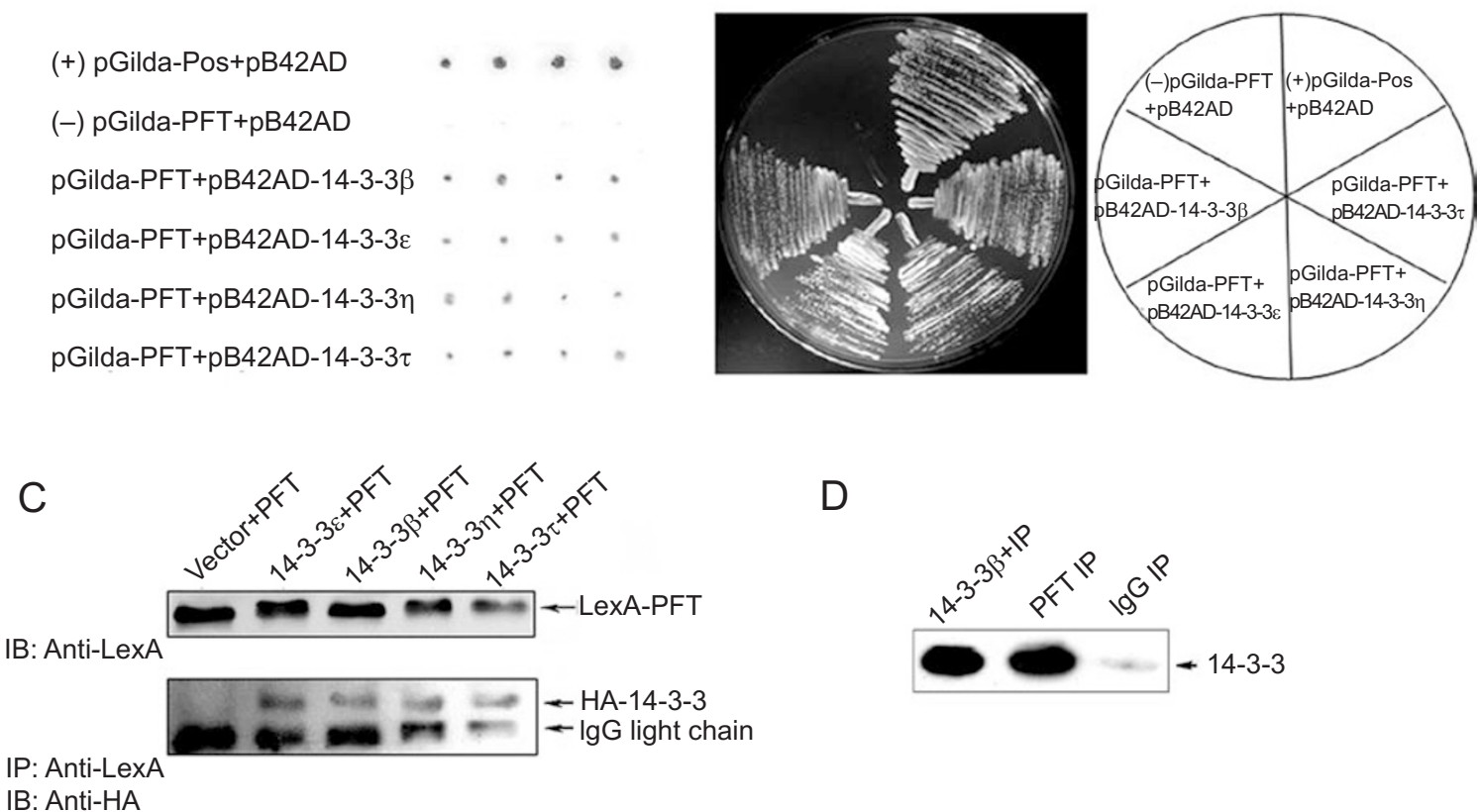

Figure 1 Interaction of hPFTAIRE1 with four 14-3-3 isoforms $(\beta, \varepsilon, \eta, \tau)$. (A) $\beta$-galactosidase activity assay. The pGilda-PFT and pB42AD-14-3-3 plasmids were co-transformed in CJY151. pGilda-Positive or pB42AD was used as a control. (B) Growth ability of transformants on minimal medium deficient in His, Trp and Leu. (C) Co-immunoprecipitation assay. The LexA-PFTAIRE1 and the HA-14-3-3 proteins were co-expressed in CJY151. The expression of LexA-PFTAIRE1 was showed as above by using antiLexA antibody in Western blot analysis. The lower panel showed the interaction between hPFTAIRE1 and 14-3-3 proteins in yeast. The tagged proteins were pulled down with anti-LexA antibody and detected in Western blot with anti-HA antibody as a probe. (D) Interaction of the hPFTAIRE1 with 14-3-3 proteins in Hela cells. The 14-3-3 proteins in Hela cells were pulled down with mouse monoclonal anti-14-3-3 $\beta$ antibody. The hPFTAIRE1 was immunoprecipitated with polyclonal anti-PFTAIRE1 antibody. The lysate was immunoprecipitated with mouse IgG and used as a control. The precipitated proteins were analysed by Western blot using anti14-3-3 $\beta$ antibody. 
precipitations of hPFTAIRE1-14-3-3 complexes were performed with anti-PFTAIRE1 or anti-14-3-3 $\beta$ antibody. The precipitated proteins were detected and analyzed by Western blotting with anti-14-3-3 $\beta$ antibody. The 14-3-3 proteins were pulled down and detected in cell lysate (Figure 1D, first lane), and the hPFTAIRE1 could be pulled down and detected to bind with 14-3-3 proteins (Figure 1D, second lane). The results suggested that the hPFTAIRE1 could interact with the 14-3-3 proteins in vivo.

The hPFTAIRE1 RHSSPSS motif is required for interaction with 14-3-3 proteins

Ligands of 14-3-3 are known to contain defined interaction motifs including two main binding consensus motifs: $\mathrm{RSxpSxP}$ and $\mathrm{Rx}_{1-2} \mathrm{Sx}_{2-3} \mathrm{~S}$. The hPFTAIRE1 contains a $\sim 140$ aa N-terminal domain, a $\sim 300$ aa kinase conserved domain in its central region, and a $\sim 30$ aa $\mathrm{C}$-terminal domain. The $\mathrm{N}$-terminal region contains two predicted nuclear localization signals (NLSs) located at residues 66 to 72 (PEDKKVR) and 113 to 119 (PKVRRHS). A putative 14-3-3 binding consensus motif RHSSPSS (117 to 120) was overlapped with the second NLS of the hPFTAIRE1 (Figure 2A).

To examine whether the putative consensus motif in the hPFTAIRE1 is required for binding of the 14-3-3 proteins,
5 residues of SSPSS from the motif was deleted by PCR based mutagenesis strategy. The fragment was inserted into vectors to produce mutated hPFTAIRE1 fusion proteins for further binding analysis. The pGilda-PFT $\Delta$ was introduced into CJY151 and the LexA-hPFTAIRE1 $\Delta$ was co-expressed with the four 14-3-3 isoforms in yeast cells and assayed in two hybrid system. The $\beta$-galactosidase activity and Leu medium growth ability analysis revealed that the hPFTAIRE1 mutant failed to interact with all four 14-3-3 isoforms (Figure 3A and 3B). The pcDNA3PFT $\Delta$-myc was transfected into Hela cells to detect the interaction between hPFTAIRE1 $\Delta$ and 14-3-3 proteins. The pcDNA3-PFT-myc was also transfected into Hela cells and used as a positive control (Figure 3C left panel). Co-IP and Western blot analysis showed that the interactions between the hPFTAIRE1 and 14-3-3 proteins were abolished by deletion of the 14-3-3 binding motif in the hPFTAIRE1 (Figure 3C right panel). The results indicate that the putative motif RHSSPSSP in the hPFTAIRE1 is required for its interaction with 14-3-3 proteins.

The $\mathrm{Ser}^{119}$ is crucial for the interaction between hPFTAIRE1 and 14-3-3 proteins

The 14-3-3 proteins bind to specific phosphoserine con-

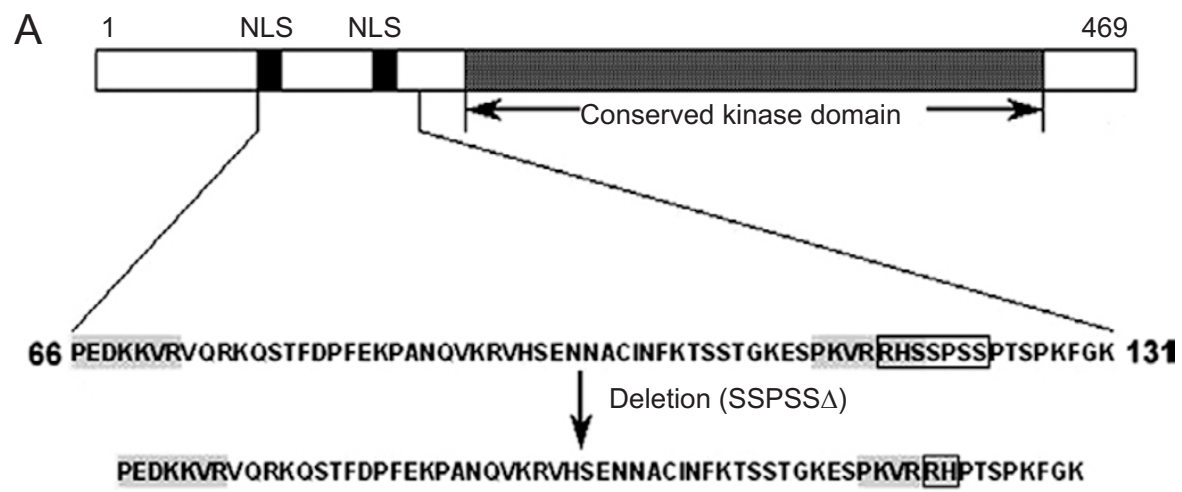

B

66 PEDKKVRVQRKQSTFDPFEKPANQVKRVHSENNACINFKTSSTGKESPKVRRHSSPSPTSPKFGK 131

$$
\begin{aligned}
& \text { hPFTAIRE1 RH } \stackrel{*}{\text { S P S S }} \\
& \text { S119AhPFTAIRE1 RH ÅS P S S } \\
& \text { S120AhPFTAIRE1 RHSÄP S S }
\end{aligned}
$$

Figure 2 Mutagenesis strategy. The hPFTAIRE1 was drawn schematicly, two predicted nuclear localization signals (NLSs) were indicated in black box and conserved kinase domain was shaded. In region of 66-131aa, the shaded amino acids indicate the two NLSs, and the boxed amino acids represent a putative 14-3-3 binding consensus sequence which overlapped with the second NLS. 5 residues of SSPSS in the 14-3-3 binding motif were deleted by using a mutagenesis kit (A). Residue of Ser ${ }^{119}$ or Ser $^{120}$ in the motif was substituted with Ala respectively (B). 
A

(+) pGilda-Pos+pB42AD

(-) pGilda-PFT+pB42AD

pGilda-PFT+pB42AD-14-3-3ع

pGilda-PFT $\Delta+$ pB42AD-14-3-3 $\beta$

pGilda-PFT $\Delta+$ pB42AD-14-3-3e

pGilda-PFT $\Delta+p B 42 A D-14-3-3 \eta$

pGilda-PFT $\Delta+p B 42 A D-14-3-3 \tau$

B
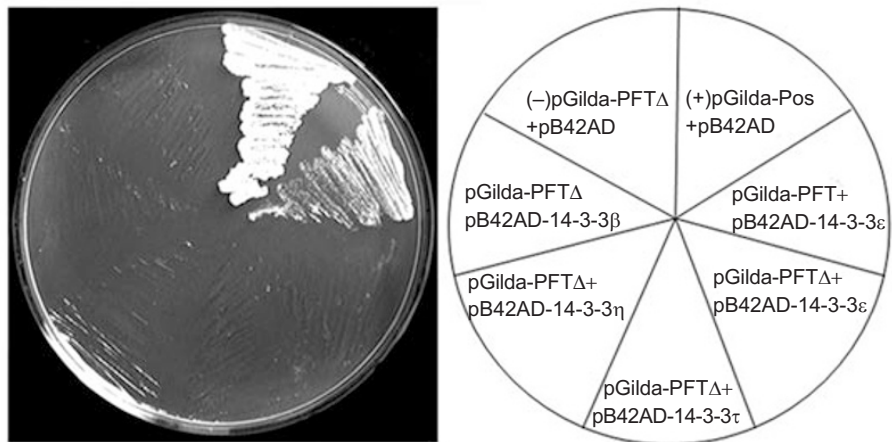

C

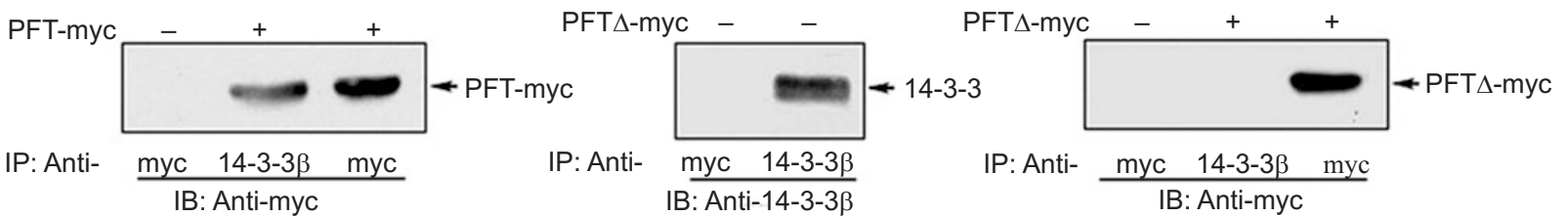

Figure 3 Examination of interaction between hPFTAIRE1 $\Delta$ and 14-3-3 proteins. (A) The pGilda-PFT $\Delta$ and pB42AD-14-3-3 plasmids were co-transformed into CJY151, co-expressed LexA-PFTAIRE1 $\Delta$ and HA-B42-14-3-3s were assayed for $\beta$-galactosidase activity. pGilda-Positive, pGilda-PFT or pB42AD was used as a control. (B) Growth ability of the transformants on minimal medium. (C) Interaction of hPFTAIRE1 $\Delta$ with 14-3-3 proteins in Hela cells. The myc tagged hPFTAIRE1 $\Delta$ was immunoprecipitated from Hela cell lysates and analysed by Western blot using anti-myc or anti-14-3-3 $\beta$ antibody. Myc tagged hPFTAIRE1 was used as a positive control.

A

(+) pGilda-Pos+pB42AD

(-) pGilda-S119APFT+pB42AD

pGilda-PFT+pB42AD-14-3-3e

pGilda-S119APFT+pB42AD-14-3-3 $\beta$

pGilda-S119APFT+pB42AD-14-3-3 $\varepsilon$

pGilda-S119APFT+pB42AD-14-3-3n

$\mathrm{B}$

pGilda-S119APFT+pB42AD-14-3-3 $\tau$
C

(+) pGilda-Pos+pB42AD

(-) pGilda-S120APFT+pB42AD

pGilda-PFT+pB42AD-14-3-3e

pGilda-S120APFT+pB42AD-14-3-3 $\beta$

pGilda-S120APFT+pB42AD-14-3-3ع

pGilda-S120APFT+pB42AD-14-3-3 $\eta$

pGilda-S120APFT+pB42AD-14-3-3 $\tau$
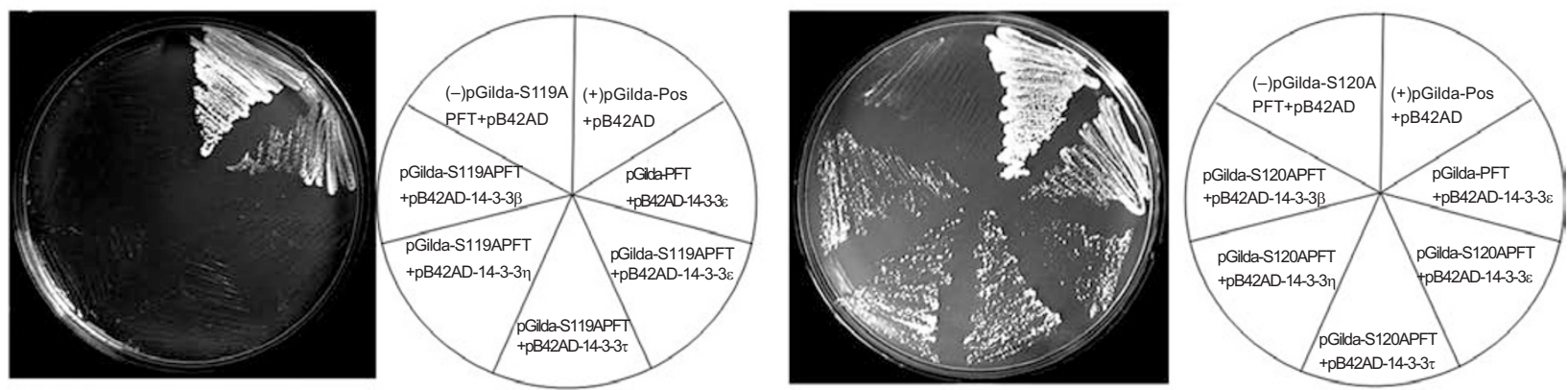

Figure 4 Interaction of S119A or S120A hPFTAIRE1 mutants with 14-3-3 proteins in yeast two hybrid system. The pGilda-S119APFT and pB42AD-14-3-3 plasmids were co-transformed into CJY151. Co-expressed LexA-S119APFTAIRE1 and HA-B42-14-3-3 were assayed in $\beta$-galactosidase activity (A) and Growth ability on minimal medium (B). LexA-S120APFTAIRE1 and HA-B42-14-3-3 were co-expressed in CJY151 for binding assay. The $\beta$-galactosidase activity $(\mathbf{C})$ and growth ability of the transformants on minimal medium (D) were examed. pGilda-Positive, pGilda-PFT or pB42AD were used as controls. 
A

\begin{tabular}{|c|c|c|}
\hline GFP & + & - \\
\hline GFP-PFT & - & + \\
\hline GFP-S119APFT & - & - \\
\hline GFP-S120APFT & - & - \\
\hline
\end{tabular}

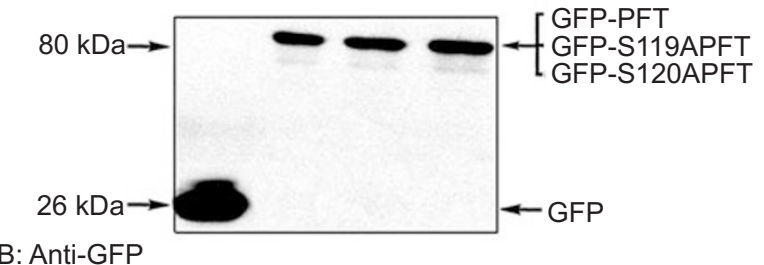

B

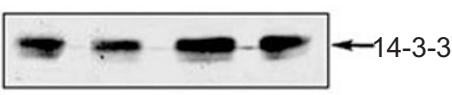

IB: Anti-14-3-3 $\beta$
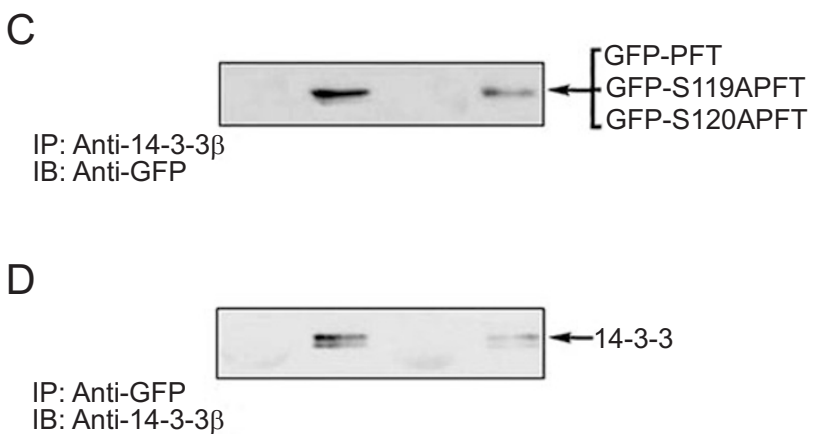

Figure 5 Interaction of hPFTAIRE1 mutants with 14-3-3 proteins in Hela cells. (A) GFP tagged hPFTAIRE1 or hPFTAIRE1 mutants were determined by using anti-GFP antibody in Western blot analysis. (B) The expression level of endogenous 14-3-3 proteins was determined with anti-14-3-3 $\beta$ antibody. (C) The 14-3-3 proteins were immunoprecipitated from the lysates and analysed by Western blot using anti-GFP antibody. (D) The GFP tagged hPFTAIRE1 and hPFTAIRE1 mutants were immunoprecipitated from the lysates and detected with anti-14-3-3 $\beta$ antibody.

taining motifs and their dimeric nature allows them to act as intra- and intermolecular bridges, effectively colocalizing proteins in a phosphorylation dependent manner [20]. To exam whether the interaction between hPFTAIRE1 and 14-3-3 proteins is phosphorylation dependent, point mutations were introduced into the 14-3-3 binding motif in the hPFTAIRE1 by PCR based mutagenesis strategy (Figure 2B). Two hPFTAIRE1 mutated frangments were inserted into vector to produce pGilda-S119APFT and pGildaS120APFT for two hybrid binding assay. The $\beta$-galactosidase activity and Leu medium growth ability analysis revealed that the S119AhPFTAIRE1 failed to interact with all four 14-3-3 isoforms (Figure 4A and 4B), however, S120AhPFTAIRE1 still could interact with 14-3-3 isoforms though the interaction was weaker than the wild type form (Figure 4C and 4D). The pEGFPC2-S119APFT and pEGFPC2-S120APFT were introduced into Hela cells to detect the interaction of two hPFTAIRE1 mutants with 14-3-3 proteins by Co-IP and Western blotting. Consistent with the two hybrid result, the mutation in $\operatorname{Ser}^{119}$ abolished the interaction between the hPFTAIRE1 and 14-3-3 proteins, but the S120A hPFTAIRE1 mutant showed a weak interaction with the 14-3-3 proteins (Figure 5C and 5D). The results indicate that both $\mathrm{Ser}^{119}$ and $\mathrm{Ser}^{120}$ in the hPFTAIRE1 contribute to its binding with 14-3-3 proteins, and the $\operatorname{Ser}^{119}$ is crucial for the interaction between them. It is possible that the interaction between hPFTAIRE1 and 14-3-3 protein may depend on phosphorylation of the Ser ${ }^{119}$.

\section{Binding to 14-3-3 does not regulate the hPFTAIRE1 intra- cellular localization}

The hPFTAIRE1 contains two predicted NLSs in its Nterminus, 184-197 ( LLKGLKHANIVLLH ) and 235-245 ( LFLFQLLRGLS ) (Figure 2A). Lazzaro et al reported that mouse PFTAIRE protein was localized both in nucleus and cytoplasm in neurons by indirect immunofluorescence. Our previous studies showed that the GFP-hPFTAIRE1 fusion was mainly localized in the cytoplasm of Hela cells [9]. 14-3-3 proteins are implicated in regulating the function and subcellular localization of numerous signaling molecules through binding to their partners. We hypothesized that the binding of 14-3-3 proteins may be involved in regulating hPFTAIRE1 subcellular distribution.

We transfected pEGFPC2-PFT $\Delta$, pEGFPC2-S119APFT and pEGFPC2-S120APFT into Hela cells and SH-SY5Y cells, pEGFPC2-PFT and pEGFPC2 were used as controls. Similar to GFP-PFTAIRE1, the ectopically expressed GFP-PFTAIRE1 $\triangle$, GFP-S119APFTAIRE1 and GFPS120APFTAIRE1 were mainly localized in cytoplasm while GFP protein distributed everywhere in Hela cells and in SH-SY5Y cells (Figure 6A and 6B). To verify that full-length instead of partial GFP- fusion protein was expressed in Hela cells, lysate from the transfected cells were immunoblotted with anti-GFP antibody. It was showed that all the expressed fusion proteins were in predicted size (Figure 5A). Deletion or point mutations in the 14-3-3 binding motif sequence did not lead the hPFTAIRE1 into nucleus indicating that binding with 14-3-3 is not required for its regulation of intracellular localization.

\section{Discussion}

The hPFTAIRE1 belongs to a member of cyclin-dependent kinase (cdk) family whose function is unknown. The kinase activity in the PFTAIRE immune complex, precipitated from a mouse brain cytosolic protein prepara- 
A

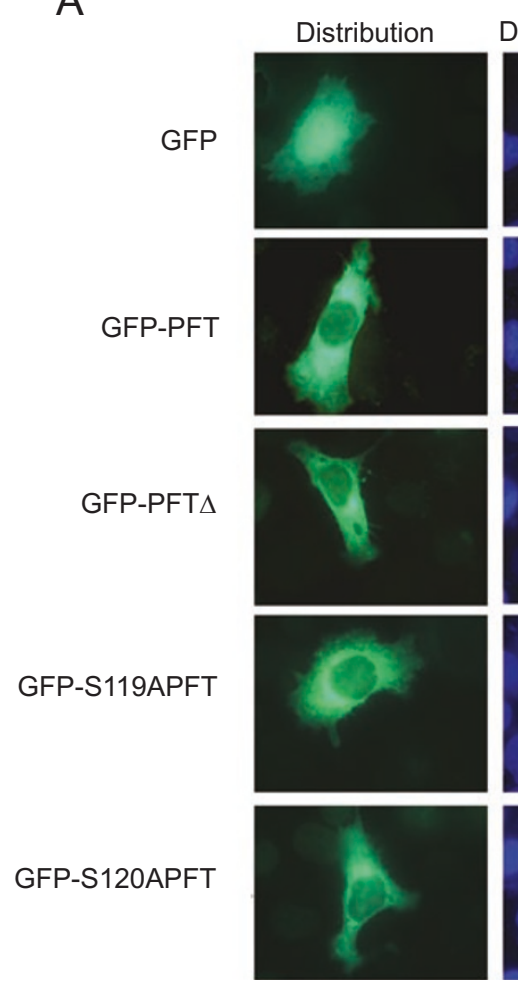

DAPI stained nuclei
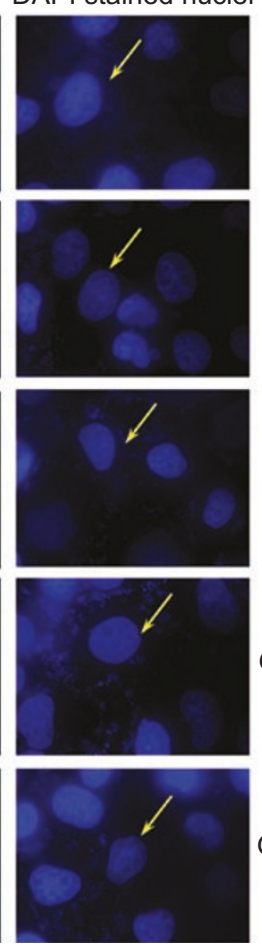

B

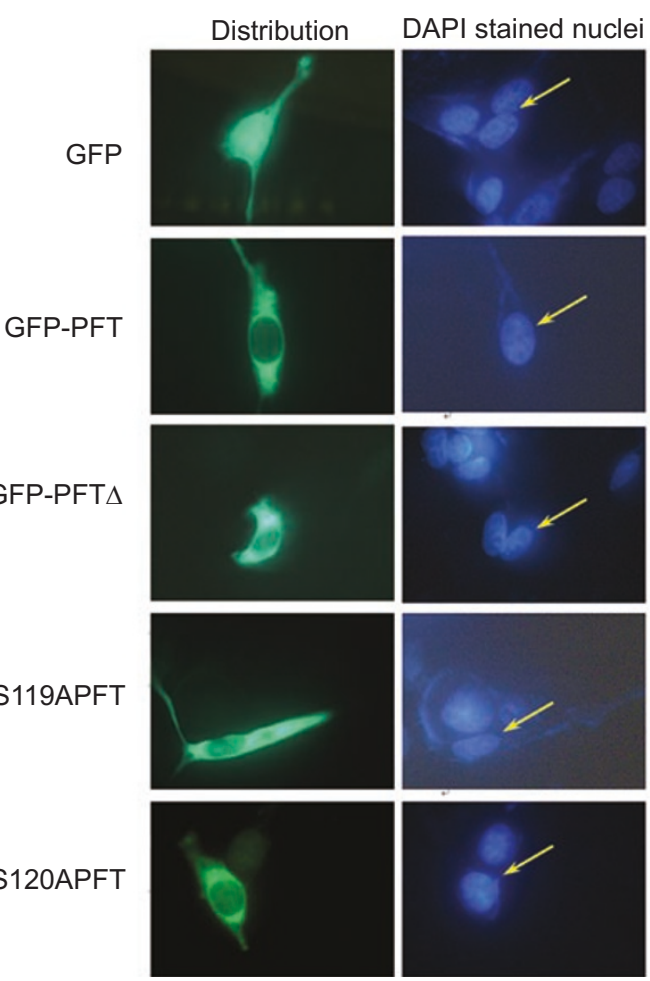

Figure 6 Subcellular localization of hPFTAIRE1 and hPFTAIRE1 mutants. (A) Hela cells were transfected with pEGFPC2, pEGFPC2PFT, pEGFPC2-PFTD, pEGFPC2-S119APFT and pEGFPC2-S120APFT respectively. (B) The plasmids were introduced into SHSY5Y cells. Green florescence of GFP, GFP-hPFTAIRE1, GFP-hPFTAIRE1 $\Delta$, GFP-S119AhPFTAIRE1 and GFP-S120AhPFTAIRE1 were observed under a florescence microscope (left panels), the nuclei were stained with DAPI (right panels).

tion, phosphorylated two specific sets of proteins of $\sim 58-60$ and 200-205 kDa. These proteins remained associated with PFTAIRE during the immunoprecipitation [7]. The immunoprecipitated PFTAIRE was not able to phosphorylate histone H1, NF-H and other common substrates, suggesting that the PFTAIRE may have a more restricted set of substrates than other cdks. In vitro-translated PFTAIRE or bacteria produced GST-PFTAIRE did not have kinase activity on their own (autophosphorylation). It is conceivable that these preparations of the PFTAIRE lacked regulatory components that may be required for its kinase activity. We identified several hPFTAIRE1 interacting proteins through two-hybrid screening. One of them we reported previously was a human septin member encoded by KIAA0202 [10]. The KIAA0202 is a 508 aa protein and has a predicted molecular mass of $58 \mathrm{kDa}$ which has similar size with the PFTAIRE substrates of $58-60 \mathrm{kDa}$ in vivo. The cytoplasmic localization of septins suggested that the KIAA0202 might act as a localizer or specific substrate for the hPFTAIRE1. In this study, we reported that four 14-3-3 isoforms $(\beta, \varepsilon$, $\eta, \tau)$ are able to interact with the hPFTAIRE1, suggesting a novel regulatory mechanism for hPFTAIRE1 may be involved. Binding with 14-3-3 proteins is involved in some nuclear proteins intracellular targeting, in case of $\mathrm{Cdc} 25$ and NFAT, 14-3-3 binding keeps them in the cytoplasm. It is also reported that binding of 14-3-3 to TTP is one of multiple mechanisms that keep TTP in the cytoplasm [22, 23]. The hPFTAIRE1 contains a putative 14-3-3 binding motif RHSSPSS overlapped with its second NLS. Deletion of 14-3-3 binding motif blocked the interaction. This result suggested that hPFTAIRE1 binds 14-3-3 proteins through its 14-3-3 binding motif RHSSPSS. It was also reported that this binding is in a phosphorylation dependent manner. Among the two binding consensus motifs: RSxpSxP and $\mathrm{Rx} 1-2 \mathrm{~S} \times 2-3 \mathrm{~S}$, the phosphorylation of one Ser is crucial for the interaction. Our results showed that substitution of Ser ${ }^{120}$ with Ala weaken the interaction, while hPFTAIRE1 Ser ${ }^{119}$ Ala completely lose its ability to interact with 14-33 proteins reflecting that phosphorylation on $\mathrm{Ser}^{119}$ may be required for the binding. All the mutants including hPFTAIRE1 $\triangle$, S119APFTAIRE1 and S120APFTAIRE1 showed a similar cytoplasmic distribution like the wild type form of the hPFTAIRE1 both in neuron cells and Hela cells, indicating that $14-3-3$ binding is not required 
for intracellular trafficking of the hPFTAIRE1. Interacting with the KIAA0202 support the suggestion of that the hPFTAIRE1 may play roles in cytoplasm. PCTAIRE1, another Cdc2-related kinase member also exhibits cytoplasmic distribution throughout the cell cycle [24]. Although our date showed that the ectopically expressed hPFTAIRE1 is mainly located in cytoplasm in neuron cells and Hela cells, mPFTAIRE1 was reported to be distributed both in nucleus and cytoplasm in neurons. Many proteins with nuclear localization signal (NLS) can be imported into nucleus mediated by importin $\alpha / \beta$ complex. Proteins containing canonical leucine-rich nuclear export signal (NES) were recognized by some export receptor complex (exportin1 or Crm1) and exported to cytoplasm [21]. A protein that shuttles between nucleus and cytoplasm usually contains a NLS that binds to an importin and a NES that binds to an exportin. The mechanism for the hPFTAIRE1 shuttling between cytoplasm and nucleus need to be further investigated.

The majority of the cells in brain and testis are not active in mitosis. The expression pattern of the hPFTAIRE1 predominantly in the brain and testis suggested that the function of the hPFTAIRE1 is different from the function of the classic Cdk family proteins that are essential for the control of mitotic cell cycle. It is also known that 14-3-3 proteins are abundantly expressed in the brain. 143-3 proteins have attracted much interest recently owing to their possible involvement in the pathophysiology of various neurological disorders. Mutations in some 14-3-3 genes lead to disruption of neuronal differentiation and synaptic plasticity in Drosophila. In humans, an interesting observation is the detection of significantly decreased levels of 14-3-3 $\gamma$ in the cortex of embryos with Down's syndrome, which may related to the impairment of neuronal differentiation, synaptic plasticity and signalling pathways. Important influence on neuronal migration has also been reported for $14-3-3 \varepsilon$. Binding of $14-3-3 \varepsilon$ to $\mathrm{Cdk} 5 / \mathrm{p} 35-$ phosphorylated Nudel maintains Nudel phosphorylation by protecting it from dephosphorylation by the serine/ threonine phosphatase PP2A. This binding has positive effects on the motor function of dynein and therefore on neuronal migration [25]. It will be interesting to explore whether hPFTAIRE1 is involved in the neuron differentiation and migration.

\section{Acknowledgements}

We thank Ms YuYing Kong for the transfection of Hela cells. This work was supported by grants from National Natural Science Foundation of China (Nos. 30400073 and 30330010).

\section{References}

1 Morgan DO. Cyclin-dependent kinases: engines, clocks, and microprocessors. Annu Rev Cell Dev Biol 1997; 13:261-291.

2 Norbury C, Blow J, Nurse P. Regulatory phosphorylation of the p34 ${ }^{\text {cdc2 }}$ protein kinase in vertebrates. EMBO J 1991; 10:33213329.

3 Meyerson M, Enders GH, Wu CL, Su LK, Gorka C. A family of human Cdc2 related protein kinases. EMBO J 1992; 11:29092917.

4 Hirose T, Tamaru T, Okumura N, Nagai K, Okada M. PCTAIRE2, a Cdc2-related serine/threonine kinase, predominantly expressed in terminally differentiated neurons. Eur J Biochem 1997; 249:481-488.

5 Besset V, Rhee K, Wolgemuth DJ. The cellular distribution and kinase activity of the Cdk family member Pctairel in the adult mouse brain and testis suggest functions in differentiation. Cell Growth differ 1999; 10:173-181.

6 Herskovits AZ, Davies P. Cloning and expression analysis of two novel PCTAIRE3 transcripts from human brain. Gene 2004; 328:59-67.

7 Lazzaro MA, Albert PR, Julien JP. A novel Cdc2-related protein kinase expressed in the nervous system. J Neurochem 1997; 69: 348-364.

8 Besset V, Rhee K, Wolgemuth DJ. The identification and characterization of expression of Pftaire-1, a novel Cdk family member, suggest its function in the mouse testis and nervous system. Mol Reprod Dev 1998; 50:18-29.

9 Yang T, Chen J. Identification and cellular localizations of human PFTAIRE1. Gene 2001; 267:165-172.

10 Yang T, Gao Y, Chen J. KIAA0202, a human septin family member, interacting with hPFTAIRE1. Acta Biochimica et Biophysica Sinica 2002; 34:520-525.

11 Tzivion G, Avruch J. 14-3-3 proteins: active cofactors in cellular regulation by serine/threonine phosphorylation. J Biol Chem 2002; 277:3061-3064.

12 Sehnke PC, DeLille JM, Ferl RJ. Consummating signal transduction: the role of 14-3-3 proteins in the completion of signalinduced transitions in protein activity. Plant Cell 2002; 14:339354.

13 Berg D, Holzmann C, Riess O. 14-3-3 proteins in the nervous system. Nat Rev Neurosci 2003; 4:752-762.

14 Rosenquist M. 14-3-3 proteins in apoptosis. Braz J Med Biol Res 2003; 36:403-408.

15 Muslin AJ, Xing H. 14-3-3 proteins: regulation of subcellular localization by molecular interference. Cell Signal 2000; 12:7039.

16 Voigt J, Liebich I, Wostemeyer J, Adam KH, Marquardt O. Nucleotide sequence, genomic organization and cell-cycle-dependent expression of a Chlamydomonas 14-3-3 gene. Biochim Biophys Acta 2000; 1492:395-405.

17 Fu H, Subramanian RR, Masters SC. 14-3-3 proteins: structure, function, and regulation. Annu Rev Pharm Toxicol 2000; 40:617647.

18 Ni J, Gao Y, Liu L, Chen J. Candida albicans Cdc37 interacts with the Crk1 Kinase and is required for Crk1 production. FEBS Lett 2004; 561:223-230.

19 Sambrook J, Russell DW. Molecular Cloning: A laboratory manual. 3rd edition. New York: Cold Spring Harbor Laboratory 
Press, 2001.

20 Muslin AJ, Tanner JW, Allen PM, Shaw AS. Interaction of 143-3 with signaling proteins is mediated by the recognition of phosphoserine. Cell 1996; 84:889-897.

21 Harel A, Forbes DJ. Importin beta: conducting a much larger cellular symphony. Mol Cell 2004; 16:319-330.

22 Kumagai A, Dunphy WG. Binding of 14-3-3 proteins and nuclear export control the intracellular localization of the mitotic inducer Cdc25. Genes Dev 1999; 13:1067-1072.

23 Yaffe MB. How do 14-3-3 proteins work? - Gatekeeper phosphorylation and the molecular anvil hypothesis. FEBS Lett 2002;
513:53-57.

24 Charrasse S, Carena I, Hagmann J, Woods-cook K, Ferrari S. PCTAIRE-1 characterization, subcellular distribution and cell cycle-dependent kinase activity. Cell Growth differ 1999; 10:611620.

25 Toyo-oka K, Shionoya A, Gambello MJ, et al. 14-3-3 epsilon is important for neuronal migration by binding to NUDEL: a molecular explanation for Miller-Dieker syndrome. Nat Genet 2003; 34:274-285.

Edited by Zhiheng Xu 